\title{
General-Purpose Programmable Photonic Chips
}

\author{
Wim Bogaerts, Mi Wang, Xiangfeng Chen, Hong Deng, Iman Zand, \\ Lukas Van Iseghem, K.P. Nagarjun, Umar Khan \\ Ghent University - IMEC, Photonic Research Group, Department of Information Technology, Gent, Belgium \\ Center for Nano-and Biophotonics (NB-Photonics), Ghent University, Gent, Belgium \\ wim.bogaerts@ugent.be
}

\begin{abstract}
Photonic chips are becoming more programmable, with connectivity that is reconfigured using electronics and software. This evolution is propelled by artificial intelligence and quantum computing applications. We will discuss more generalpurpose circuits that could be deployed in more diverse applications, similar to general-purpose programmable electronics.

Photons are the world's favorite data carrier, in the form of fiber optic links. But more and more we see that photonic information is handled on the surface of a chip, and not just for data transport, but also for processing. While photonic integrated circuits (PIC) were mostly limited to very specific functions (e.g. transceivers) the technology is slowly finding its way into diverse application spaces.
\end{abstract}

This is supported by rapidly maturing PIC technology platforms in a variety of material systems, such as III-V semiconductors, silicon, or silicon nitride. Fabricated with similar semiconductor technologies as electronic chips, these PIC platforms support dense integration of 100s or 1000s of optical building blocks on a chip. When these building blocks include electrically tunable elements, the behaviour of the chip can be actively manipulated. As a result, static photonic integrated circuits have gradually become more tunable, where the performance or the functionality can be adjusted at run time. Of course, this requires the integration of the photonic circuit with an electronic driver circuit.

In the past 5 years, the widespread availability of tunable elements on a photonic chip has given rise to so-called 'programmable' photonic circuits. In a programmable PIC, the paths of the light are not predefined. Instead, the circuit consists of a mesh of waveguides connected together with $2 \times 2$ optical gates, which consist of a tunable $2 \times 2$ coupler (the on-chip equivalent of a free-space $2 \times 2$ optical beamsplitter) and a phase shifter (or an equivalent optical subcircuit). Such waveguide meshes are sketched in Fig. 1. By tuning the coupling coefficients of the gates, the light can be distributed over the different waveguide paths on the chip, and with the phase shifters the interference between these different paths can be controlled. The result is massive multi-path interferometer that can be controlled by the user at run time.

We can identify two main classes of programmable waveguide meshes, as shown in Fig. 1 [1]. In forward-only meshes the light propagates in one direction from a set of input ports to a set of output ports. The optical gates control how

This work is supported by the European Union grants 780283 (MORPHIC) and 725555 (PhotonicSWARM), and by the Flemish Research Foundation (FWO-Vlaanderen) through grant G020421N (GRAPHSPAY)

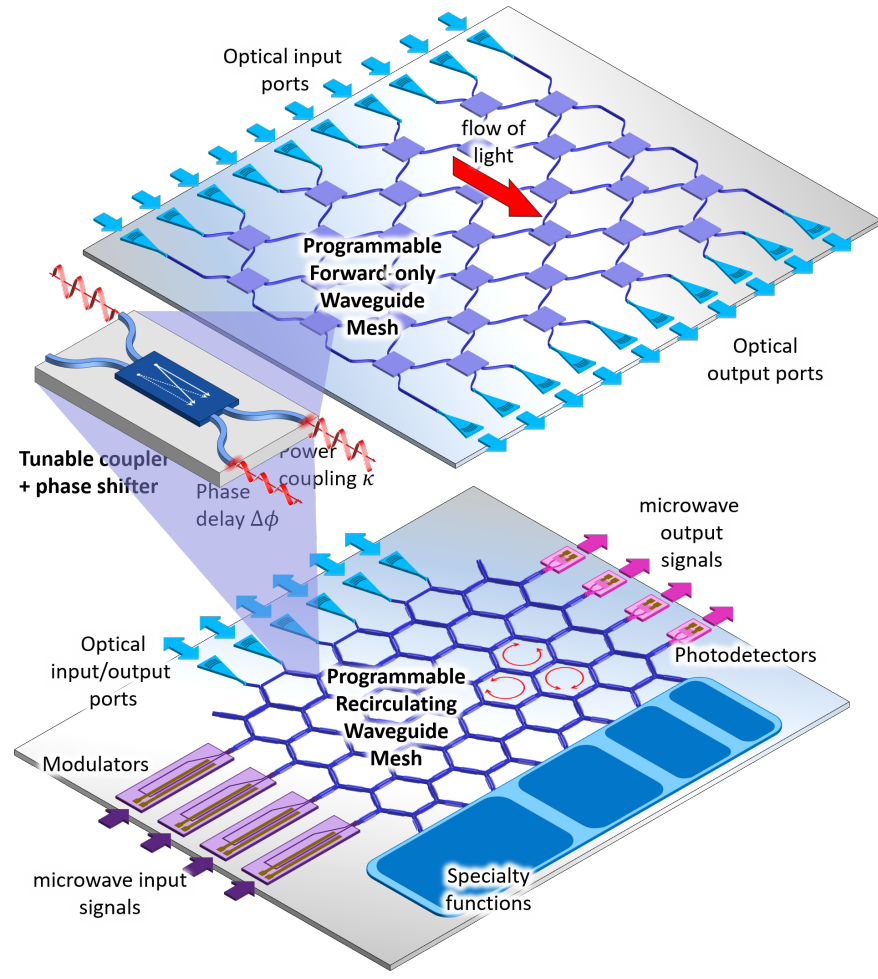

Fig. 1. Programmable Photonic Circuits. Top: Forward-only circuit. Bottom: recirculating mesh. Both consist of $2 \times 2$ optical gates.

the waveguides are coupled, where the optical fields in the output ports are a linear combination of the fields in the input ports [2]. Such an operation effectively performs a complex matrix-vector multiplication of the amplitudes/phases in the input ports with a matrix determined by the configuration of the mesh. Such operations are especially useful for machine learning algorithms [3] but also for quantum information processing, where the qubits are encoded in the amplitude and phase of single photons propagating through the mesh [4].

While there are different arrangements of forward-only meshes that can be used to construct an arbitrary linear transformation between the input and output ports, the functionality of a forward-only mesh is inherently limited to the mapping of a preassigned set of input ports onto output ports. More complicated photonic functions, even if they are purely passive, are more difficult to implement: wavelength filters, reflectors or resonators. 


\begin{tabular}{|c|c|c|c|c|c|c|}
\hline developer kit & \multicolumn{4}{|c|}{ programming environment } & \multicolumn{2}{|c|}{$\begin{array}{l}\text { development } \\
\text { board }\end{array}$} \\
\hline programming & \multicolumn{2}{|c|}{$\begin{array}{l}\text { reusable programming } \\
\text { blocks (IP) }\end{array}$} & \multicolumn{2}{|c|}{$\begin{array}{l}\text { routing and filter } \\
\text { synthesis algorithms }\end{array}$} & \multicolumn{2}{|c|}{$\begin{array}{l}\text { software interface to the } \\
\text { individual elements }\end{array}$} \\
\hline control strategies & \multicolumn{2}{|c|}{$\begin{array}{l}\text { global control } \\
\text { algorithms }\end{array}$} & \multicolumn{2}{|c|}{$\begin{array}{c}\text { model generation and } \\
\text { parameter extraction }\end{array}$} & \multicolumn{2}{|c|}{ local control strategies } \\
\hline packaging & \multicolumn{4}{|c|}{$\begin{array}{l}1000 \text { s of DC connections } \\
\text { (flip-chip / 3D stacking / interposer) }\end{array}$} & $\begin{array}{l}\text { RF waveguides } \\
\text { and connectors }\end{array}$ & $\begin{array}{l}\text { fiber array } \\
\text { interface }\end{array}$ \\
\hline $\begin{array}{l}\text { digital electronics } \\
\text { ( } \mu \text { controller, FPGA) }\end{array}$ & $\begin{array}{l}\text { high-level } \\
\text { control loops }\end{array}$ & \multicolumn{2}{|c|}{ PWM or DAC } & $A D C$ & $\begin{array}{l}\text { RF self-test } \\
\text { functions }\end{array}$ & \\
\hline analog electronics & $\begin{array}{c}\text { fast } \\
\text { control loops }\end{array}$ & \multicolumn{2}{|c|}{$\begin{array}{c}\text { current or } \\
\text { voltage drivers }\end{array}$} & $\begin{array}{l}\text { readout } \\
\text { amplifiers }\end{array}$ & \multicolumn{2}{|c|}{$\begin{array}{c}\text { RF drivers } \\
\text { trans-impedance } \\
\text { amplifier (TIA) }\end{array}$} \\
\hline photonic chip & $\begin{array}{c}\text { mesh } \\
\text { achitecture }\end{array}$ & \multicolumn{2}{|c|}{$\begin{array}{c}\text { phase shifters } \\
\text { couplers }\end{array}$} & $\begin{array}{l}\text { power / phase } \\
\text { monitors }\end{array}$ & $\begin{array}{l}\text { modulators } \\
\text { detectors }\end{array}$ & $\begin{array}{l}\text { optical IO: } \\
\text { fiber couplers }\end{array}$ \\
\hline
\end{tabular}

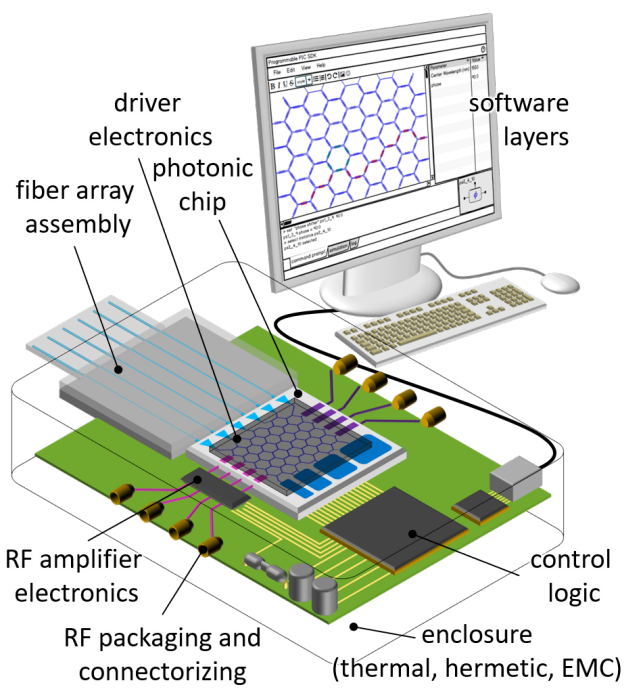

Fig. 2. The technology stack of programmable photonics [5]

The second type of waveguide mesh, which we call a recirculating mesh, overcomes many of these limitations. The waveguides are now arranged in loops connected with the same type of $2 \times 2$ optical gates [6]. The unit cells of these meshes can be triangular, square or hexagonal, and light can circulate in clockwise or counterclockwise direction [7]. This scheme effectively connects all exposed waveguides on the periphery, so all ports can act as either input or output.

Because of this omnidirectional propagation, recirculating meshes are more flexible in the definition of optical paths. It is possible to implement paths with different optical lengths, which opens the gate to implement interferometric wavelength filters. The waveguide loops themselves can also be used as ring resonators or loop mirrors.

Because all ports can be considered equivalent, it is possible to use them to attach other functional devices to the mesh, such as high-speed modulators, photodetectors, light sources or other specialized functions such as long, low-loss delay lines. With this scheme, the recirculating mesh can be configured into different circuits connecting these active functions together. This capability, combining programmable connectivity with all the other functions of classical photonic integrated circuits, makes the recirculating meshes a generalpurpose programmable PIC technology. For example, a recirculating mesh can be configured as an optical transceiver connecting a modulator and detector to input and output fibers. Because the mesh also has full phase control, a coherent IQ modulator and coherence receiver can also be constructed, and it is even possible to implement wavelength filters for wavelength division multiplexing. The same filters could be used to implement a sensor readout system or spectrometer. The high-speed modulators and detectors can also be used to translate microwave signals into the optical domain so filtering or frequency conversion can be programmed in the waveguide mesh [6].
The path towards general-purpose PICs has not yet fully materialized. To realize its full potential, all elements of the technology stack (Fig. 2) need to be in place, starting from the photonic chip technology all the way to the software layers that allow the end user to access the chip functions.

A general-purpose photonic circuit introduces many advantages that we are familiar with from the electronics world. Off-the-shelf availability, together with a software interface, enables fast prototyping of new functionality, and when integrated into products, offer a path for fixes and upgrades. Also, a general-purpose chip can address more diverse markets and therefore be fabricated into larger volumes [5]. Given that photonic chip volumes are still orders of magnitude lower than those of electronics, this aggregation of production volume spreads many non-recurrent engineering costs over a larger user group. This, in turn, lowers the barriers for new entrants in the field of photonics.

\section{REFERENCES}

[1] W. Bogaerts, D. Pérez, J. Capmany, D. A. Miller, J. Poon, D. Englund, F. Morichetti, and A. Melloni, "Programmable photonic circuits," Nature, vol. 586, no. 7828, pp. 207-216, 2020.

[2] D. A. B. Miller, "Self-configuring universal linear optical component [Invited]," Photonics Research, vol. 1, no. 1, p. 1, 2013.

[3] Y. Shen, N. C. Harris, S. Skirlo, M. Prabhu, T. Baehr-jones, M. Hochberg, X. Sun, S. Zhao, H. Larochelle, D. Englund, and M. Solja, "Deep learning with coherent nanophotonic circuits," Nature Photonics, vol. 11, no. June, pp. 441-447, 2017.

[4] J. Carolan, C. Harrold, C. Sparrow, E. Martin-Lopez, N. J. Russell, J. W. Silverstone, P. J. Shadbolt, N. Matsuda, M. Oguma, M. Itoh, G. D. Marshall, M. G. Thompson, J. C. F. Matthews, T. Hashimoto, J. L. O'Brien, and A. Laing, "Universal linear optics," Science, vol. 349, no. 6249, pp. 711-716, 2015.

[5] W. Bogaerts and A. Rahim, "Programmable Photonics: An Opportunity for an Accessible Large-Volume PIC Ecosystem," IEEE Journal of Selected Topics in Quantum Electronics, vol. 26, no. 5, p. 1, 2020.

[6] J. Capmany, I. Gasulla, and D. Pérez, "Microwave photonics: The programmable processor," Nature Photonics, vol. 10, no. 1, pp. 6-8, 2016.

[7] D. Pérez, I. Gasulla, J. Capmany, and R. A. Soref, "Reconfigurable lattice mesh designs for programmable photonic processors," Optics Express, vol. 24, no. 11, p. 12093, 2016. 